\title{
Modulus of Natural Rubber Cross-Linked by Dicumyl Peroxide II. Comparison With Theory
}

\author{
Lawrence A. Wood \\ Institute for Materials Research, National Bureau of Standards, Washington, D.C. 20234
}

(September 22, 1972)

\begin{abstract}
Thermodynamics and molecular considerations are applied to an examination of the equation $G=S(f p+B) T+H(f p+B)+A=5.925 \times 10^{-3}(f p-0.45) T+0.0684(f p-0.45)+2.70$, found experimentally in Part I. $G$ is the shear modulus in Mdyn $\mathrm{cm}^{-2}$ at a temperature $T$ for natural rubber crosslinked by adding $p$ parts of dicumyl peroxide per hundred of rubber (phr) and heating until a fraction $f$ of the peroxide is decomposed. $G^{*}$, the energy component of the modulus, is $H(f p+B)+A$. The ratio $G^{*} / G$ decreases from 1.00 at the gel point $(f p=0.45 \mathrm{phr})$ to 0.5 near $2 \mathrm{phr}$ and to 0.09 at 23.8 phr. The modulus $G$ is related to $\nu_{e}$, the number of moles of effective sub-chains per $\mathrm{cm}^{3}$, by the equation $G-G^{*}=\nu_{e} R T$ where $R$ is the gas constant. If each molecule of decomposed dicumyl peroxide of molecular weight $M_{\boldsymbol{d}}$ produces one cross-link in the rubber of specific volume $\bar{v}_{r}$, then it is predicted that $S=2 R\left(100 M_{d} \bar{v}_{r}\right)^{-1}=5.5535 \times 10^{-3} \mathrm{Mdyn}^{\mathrm{cm}^{-2}} \mathrm{phr}^{-1} \mathrm{~K}^{-1}$, as compared with the experimental value $5.925 \times 10^{-3}$. Theory gives no prediction of the values of $A$, or of $H$. The gel point may be located experimentally as the point where the slope of the modulus-temperature relation is zero. The value of $G$ at the gel point is the energy component $G^{*}$. The experimental value of $f p$ at the gel point permits a calculation of the molecular weight of the rubber before cross-linking as 193,000. The results afford a very satisfactory confirmation of the essential validity of the statistical theory of rubber elasticity in its simplest form, if due regard is paid to $G^{*}$, the energy component of the modulus.
\end{abstract}

Key words: Cross-linking of rubber; dicumyl peroxide; elasticity theory of rubber; entropy component; gel point; modulus of rubber; rubber elasticity; statistical theory of rubber elasticity; thermodynamics of rubber elasticity.

\section{Introduction}

In a previous paper, Part I [1] ${ }^{1}$, time-independent experimental observations were reported which could be represented by the following four-constant equation:

$$
\begin{aligned}
G= & S(f p+B) T+H(f p+B)+A \\
=5.925 \times 10^{-3}(f p-0.45) T+0.0684(f p-0.45) & +2.70
\end{aligned}
$$

$S, B, H$, and $A$ are constants having the values shown, while $G$ is the shear modulus (in Mdyn $\mathrm{cm}^{-2}$ or 0.1 $\mathrm{MNm}^{-2}$ ) at a temperature $T$ (in kelvins) for natural rubber cross-linked by adding $p$ parts of dicumyl peroxide per hundred of rubber (phr), and heating until a fraction $f$ of the peroxide was decomposed. The range of values of $f p$ investigated was from 0 to 23.8 phr at temperatures from 223 to $373 \mathrm{~K}$. The shear modulus is defined as the limit of the ratio of shear stress to the corresponding strain as the stress and strain approach zero.

\footnotetext{
${ }^{1}$ Figures in brackets indicate the literature references at the end of this paper.
}

The experimental work described in Part I involved observations of the indentation of a rubber sheet by a rigid ball. Consequently, the deformation was complex, involving extension, compression, and shear in different portions of the sheet. However all deformations were kept so small that no extrapolation was required to obtain the modulus. At these small deformations the relations between stress and strain were linear. Previous work discussed in Part I has demonstrated that the values of $G$ obtained on dicumyl peroxide vulcanizates by the indentation method give good agreement with those obtained by direct measurements of shear modulus with a torsion pendulum or those given by the proper extrapolation of simple extension measurements.

Figure 1 shows the modulus-temperature relations exhibited by this equation, while figure 2 shows the plot of modulus against $f p$. In each case the shaded area shows the range of temperature covered by our experimental observations. It should be noted that extending the curves to $0 \mathrm{~K}$ involves a linear extrapolation over a range of lower temperatures about 50 percent more than the experimental range itself. However, the figure is not intended to imply that observations at temperatures below the indicated experi- 


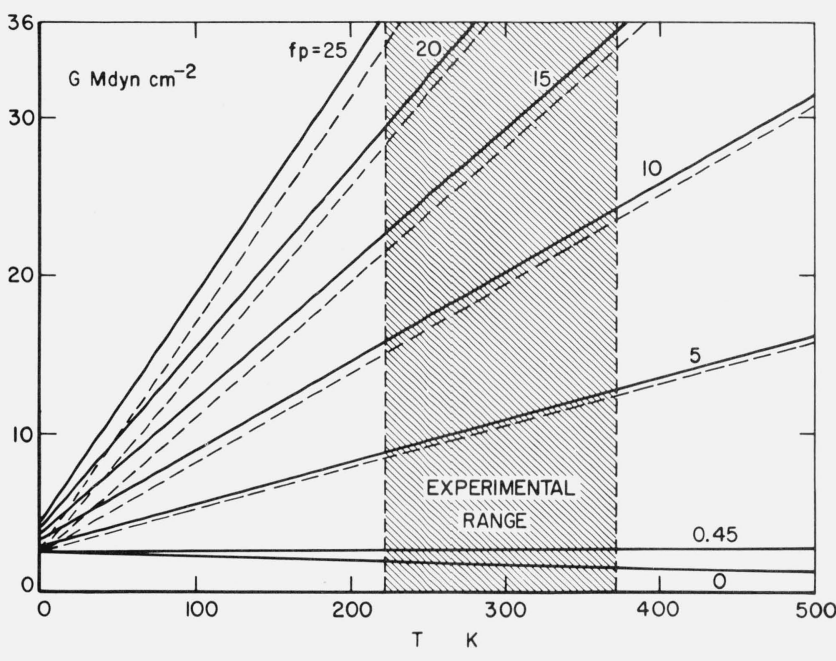

Figure 1. Modulus as a function of temperature for different amounts of decomposed dicumyl peroxide.

Continuous line represents eq (1.1)

Dashed line represents eq (1.2)

mental range would conform to the lines shown. In fact the data in Part I clearly show a completely different relation at temperatures approaching the glass transition. The general qualifications and limitations of eq (1.1) in representing experimental data are outlined in Part I. They will not be given further consideration in the present paper.

In figure 2 the range of values of $f p$ shown is limited to those below $5 \mathrm{phr}$ merely in order to present more detail at low degrees of crosslinking, but the lines shown were determined by observations over the whole range of values of $f p$.

The dashed curves represent the corresponding relations for the simpler equation

$$
G=5.925 \times 10^{-3}(f p-0.45) T+2.70
$$

The difference between the solid and dashed curves shows the effect of omitting the middle term of the right hand side of eq (1.1). When $f p=0.45$ the value of $G$ at all temperatures is given as $2.70{\mathrm{Mdyn} \mathrm{dm}^{-2}}^{-2}$ by both equations. For $f p=0$ the difference between the values given by the two equations is only 0.03 Mdyn $\mathrm{cm}^{-2}$ and so is not perceptible on the scale of ordinates used in figure 1 .

After pointing out the thermodynamic implications of eq (1.1) the present paper is devoted to a discussion of the predictions of the simplest form of the theory of rubber elasticity and a quantitative comparison of these predictions with the experimental observations. The aim has been to outline the general features of the field.

\section{Thermodynamics: Entropy and Energy}

The force involved in stretching a rubber strip can be resolved into components arising from changes in

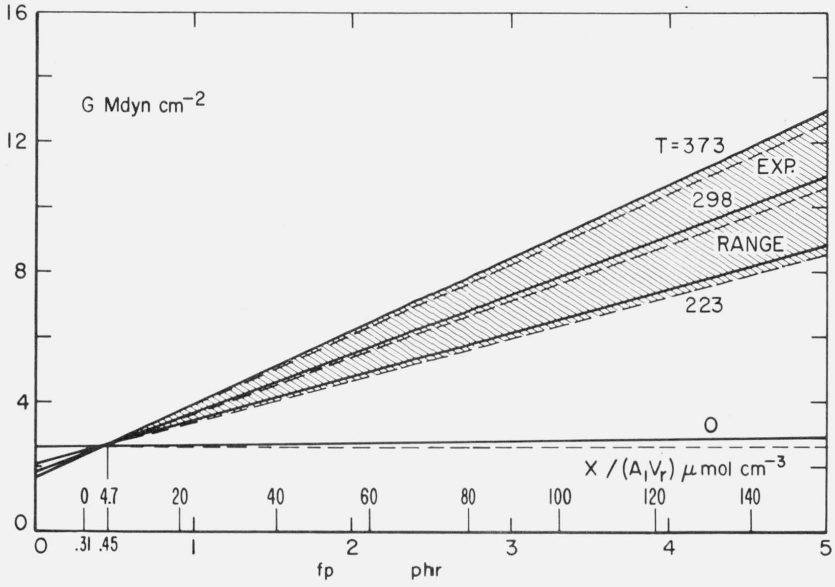

FigUre 2. Modulus as a function of amount of decomposed dicumyl peroxide for different temperatures.

Continuous line represents eq (1.1)

Dashed line represents eq (1.2).

The upper abscissa scale shows the expected density of cross-links in $\mu \mathrm{mol} \mathrm{cm}{ }^{-3}$, as calculated from eq (3.6).

entropy and internal energy. Extensive discussions [2-5] have pointed out the difference between the resolution obtained at constant pressure and that at constant volume.

Since an increase of volume is observed when rubber is stretched at constant pressure [6-8], there is a corresponding increase of internal energy. Consequently the energy component of the force at constant pressure exceeds that at constant volume by the force corresponding to the amount of work required to increase the volume. The entropy component is necessarily smaller by the same amount, if the total force is kept the same.

The theory of rubber elasticity usually places emphasis on the configurational aspects of the problem, and so normally requires the resolution to be made at constant volume in order to eliminate that portion of the energy component arising from volume change [9].

The modulus $G$ studied in the present investigation can be resolved in an analogous manner into components arising from entropy and internal energy changes $[10,11]$.

However, Shen $[14,15]$ has pointed out that the shear modulus should be independent of the applied hydrostatic pressure and no distinction need be made between measurements of $G$ at constant pressure and those at constant volume. This is an important advantage of dealing with the modulus rather than the force.

In approximate confirmation of this expectation, Paterson [12] by direct observation found that the modulus of a pure-gum natural rubber vulcanizate increased by only about 30 percent as the pressure was raised from atmospheric to 4000 bars $\left(1\right.$ bar $=10^{6} \mathrm{dyn}$ $\left.\mathrm{cm}^{-2}=10^{5} \mathrm{Nm}^{-2}=10^{5} \mathrm{~Pa}\right)$. The compressibility of natural rubber cross-linked with a small amount of dicumyl peroxide has been measured as $51.4 \times$ 
$10^{-6} \mathrm{bar}^{-1}[13]$. Utilizing this value one can calculate that an external pressure of only about 2 bars would suffice to reduce to zero the volume increase of about 0.01 percent observed [6-8] on stretching rubber to an elongation of 100 percent. Consequently it seems quite certain that in an experiment where the pressure would be systematically increased to keep the volume constant, the change in modulus would be less than the experimental uncertainty of measurement.

The resolution of modulus in the present investigation can be seen by an examination of figure 1 and eq (1.1).

Equation (1.1) may be written as

$$
G=T(\partial G / \partial T)+G^{*}
$$

where

$$
\partial G / \partial T=S(f p+B)
$$

and

$$
G^{*}=A+H(f p+B)
$$

$G^{*}$ represents the component of $G$ arising from internal energy changes. The term $T(\partial G / \partial T)$, proportional to the temperature, represents the component of $G$ arising from increase of entropy as the temperature is raised above $0 \mathrm{~K}$. Of course this entropy component can also be written as $\left(G-G^{*}\right)$.

Equation (2.1) can also be written in the form

$$
G^{*} / G=1-(T / G)(\partial G / \partial T)=1-\partial \ln G / \partial \ln T
$$

$G^{*}$ can be noted as the intercept at $0 \mathrm{~K}$ for each line in figure 1. Equation (2.3) shows that $G^{*}$ is mainly determined by the value of the constant $A$, and would be equal to it, were the constant $H$ to be taken as zero, as in eq (1.2), represented by the dashed lines of figure 1.

$G_{0}$ the intercept of each line in figure 2, corresponding to no added cross-links, should be carefully distinguished from $G^{*}$, the intercept in figure 1. In fact, from eqs (1.1) and (2.3) the relation between them is found to be

$$
G_{0}=G^{*}+S B T=2.67-2.666 \times 10^{-3} T
$$

The energy and entropy components as well as the ratios of ordinates $G^{*} / G$ in figure 1, as calculated from eqs (2.3) and (1.1) at 298.15 K, are shown in table 1.

The energy component is strongly predominant at low degrees of cross-linking. However as $f p$ is increased from 0 to $25 \mathrm{phr}$ the intercept term $G^{*}$ remains in the range 2.67 to $4.38 \mathrm{Mdyn} \mathrm{cm}^{-2}$ while the entropy component increases steadily with increased crosslinking. It becomes greater than $G^{*}$ at $298.15 \mathrm{~K}$ only when $f p$ exceeds about $2 \mathrm{phr}$ and the ultimate elongation has fallen below 200 percent. At the higher degrees of cross-linking (especially above $5 \mathrm{phr}$ ) the ultimate elongation and tensile strength are so low that the material can hardly be considered a very useful rubber. It is not clear why these results differ from those of Rehage, Schäfer, and Schwarz [11], who reported that the value of $G^{*} / G$ increased from about
TABLE 1. Energy and entropy components at $298.15 \mathrm{~K}$

$$
G^{*}=0.0684 f p+2.67 ; G=1.835 f p+1.87
$$

\begin{tabular}{l|c|c|c|c}
\hline \hline$f p$ & $\begin{array}{c}G^{*} \\
\text { Mdyn cm }\end{array}$ & $\begin{array}{c}G \\
\text { Mdyn } \mathrm{cm}^{-2}\end{array}$ & $G^{*} / G$ & $\begin{array}{c}G-G^{*} \\
\text { Mdyn } \mathrm{cm}^{-2}\end{array}$ \\
\hline 0 & 2.67 & 1.87 & $\ldots \ldots \ldots \ldots \ldots \ldots \ldots \ldots . .$. & $\ldots \ldots \ldots \ldots$ \\
0.45 & 2.70 & 2.70 & 1.000 & 0 \\
1 & 2.74 & 3.71 & 0.739 & 0.97 \\
2 & 2.81 & 5.54 & .507 & 2.73 \\
3 & 2.88 & 7.38 & .390 & 4.50 \\
4 & 2.94 & 9.21 & .319 & 6.27 \\
5 & 3.01 & 11.05 & .272 & 8.04 \\
10 & 3.35 & 20.22 & .166 & 16.87 \\
15 & 3.70 & 29.40 & .126 & 25.70 \\
20 & 4.04 & 38.57 & .105 & 34.53 \\
25 & 4.38 & 47.75 & .092 & 43.37 \\
\hline
\end{tabular}

0.20 to 0.40 as the cross-linking was increased from zero to a value corresponding to $f p=2 \mathrm{phr}$.

Considerable attention has been given by previous investigators $[14-26]$ to a ratio they have denoted as $f_{e} / f$, where $f_{e}$ is the energy component of the force and $f$ (in contradistinction to the $f$ used in this paper) is the total value of the force required to maintain a certain elongation at constant volume. Shen and co-workers [14, 15, 27] by using the equaiion of state given by the current statistical theory of rubber elasticity have derived equations showing $f_{e} / f$ to be less than $G^{*} / G$ by an amount $(1 / 3) \beta T$ where $\beta$ is the volume thermal expansivity. This difference amounts to only 0.067 near $300 \mathrm{~K}$. We prefer to express results in terms of $G^{*} / G$, so that no assumptions regarding the form of stress-strain relation (or equation of state) are required.

Our work yielding the value of $G^{*} / G$ is of course limited to very small deformations. Consequently it gives no information on the disputed question of whether $f_{e} / f$ varies with deformation. Several observers $[21,23,24,26,27]$ had reported that $f_{e} / f$ at a fixed amount of cross-linking increased rapidly as the deformation approached zero, rising from values near 0.2 to 0.6 or more. However more recent papers by two of the authors and others $[9,14,15,25,28,29]$ have stated that the ratio is independent of the deformation, as required by theoretical considerations.

\section{Predictions of the Theory of Rubber Elasticity}

\subsection{Cross-Linking}

Modern ideas regarding cross-linking and its effect on modulus are summarized in the book by Meares [30] along the lines of the earlier developments given in the books of Flory [31] and Treloar [32]. We shall follow mainly the treatment and symbolism of Meares.

At this stage we shall limit ourselves to the very simplest assumptions, realizing that these may represent an over-simplification of the problem. In a later paper we shall consider limitations and qualifications which might be necessary and discuss the effects of more complex assumptions. Meares [33] has sum- 
marized many of the efforts to obtain more satisfactory results by more sophisticated assumptions.

Consider a quantity of unvulcanized rubber having a mass $m_{r}$, a volume $V_{r}$ and a specific volume $\bar{v}_{r}=V_{r} / m_{r}$. Let the number of rubber molecules in the system be denoted as

$$
N=A_{1} m_{r} / M=\left(A_{1} V_{r}\right) /\left(M \bar{v}_{r}\right)
$$

where $A_{1}$ is the Avogadro Constant (the number of molecules in one mole), and $M$ is the effective molecular weight of the unvulcanized rubber (here assumed to be monodisperse).

Suppose that in some manner (as by the reaction with dicumyl peroxide) a certain number of cross-links $X$ are formed in this rubber without appreciable change of volume or mass of the system. The number of subchains formed will be twice this value, since each additional cross-link gives rise to 2 more sub-chains. Sub-chains are called "strands" in the terminology used by Ferry and his collaborators.

Sub-chains which are at each end of a rubber molecule will not be effective in maintaining stresses, since they are not part of the network being formed. Thus $2 N$ sub-chains are ineffective dangling loose ends, at all degrees of cross-linking. This number should be subtracted from $2 X$ the total number of sub-chains formed, in order to obtain the number of effective or active sub-chains, that is, those capable of supporting a stress. As long as $X$ is less than $N$ some molecules can not be included, and will not be supporting their share of the stress.

Assuming the accuracy of this correction and neglecting other inactive sub-chains (such as loops for example or molecules not a part of the network), if any, one obtains $\nu_{e}$, the number of moles of active subchains per unit volume of uncured rubber.

$$
\nu_{e}=2 X /\left(A_{1} V_{r}\right)-2 /\left(M \bar{v}_{r}\right)
$$

\subsection{Cross-Linking by Dicumyl Peroxide}

The cross-linking in the present work was produced by free radicals resulting from the decomposition of dicumyl peroxide of molecular weight $M_{d}(=270.38)$. The compounding involved the addition of $p$ parts of peroxide of mass $m_{d}$ to each hundred parts of rubber of mass $m_{r}$. Thus,

$$
p=100 m_{d} / m_{r} .
$$

Some of the peroxide is wasted in reactions with impurities in the rubber $[34,35]$. Since the amount wasted $m_{0}$ would be expected to be proportional to the amount $m_{r}$ of rubber, let us define a constant $w$ such that

$$
w=100 m_{0} / m_{r} .
$$

The remainder of the dicumyl peroxide $\left(m_{d}-m_{0}\right)$, after decomposition, is available for cross-linking the rubber.
Each molecule of the remaining peroxide is assumed to give rise to one cross-link, after decomposition. Thus the total number of cross-links formed

$$
X=A_{1} f\left(m_{d}-m_{0}\right) / M_{d}
$$

where $f$ is the fraction of dicumyl peroxide decomposed during the time of vulcanization. From eqs (3.3)-(3.5) one can express the number of crosslinks formed as

$$
X=\left(A_{1} V_{r} / 100 M_{d} \bar{v}_{r}\right)(f p-f w) .
$$

This equation is used as the basis for the upper abscissa scale shown in figure 2 .

The number of cross-links formed per rubber molecule is then obtained by dividing by eq (3.1).

$$
X / N=\left(M / 100 M_{d}\right)(f p-f w)
$$

When the value of $X$ from eq (3.6) is inserted in eq (3.2) we have

$$
\nu_{e}=\left[2 / \bar{v}_{r}\right]\left[\left(1 / 100 M_{d}\right)(f p-f w)-1 / M\right] .
$$

\subsection{Variation of Modulus With Cross-linking}

A complete network including all the rubber molecules is possible only when there is at least one cross-link for each molecule, that is when $X \geqslant N$, as already stated. When the number of cross-links is increased beyond the point where $X=N$, the fundamental proposition of the statistical theory of rubber elasticity states that the formation of additional subchains will give rise to a corresponding proportional increase in modulus to a modulus value $G$ such that

$$
G-G^{*}=\nu_{e} R T
$$

where $R$ is the gas constant, $T$ the temperature in kelvins, and $G^{*}$ the modulus of the network at $0 \mathrm{~K}$, already defined as the energy component of the modulus in section 2, and utilized in eqs (2.1) and (2.3). It can be seen from eq (3.9) that $G^{*}$ should be also the modulus at all temperatures when $v_{e}=0$, namely before any effective subchains have been formed. No attempt will be made in this paper to calculate the value of $G^{*}$ from molecular constants, but it will be assumed to remain constant as the temperature is varied.

It is expected that eq (3.9) will be subject to experimental verification only above the glass transition temperature $T_{g}$. The present theory likewise will give no consideration to the case where $X<N$, since the theory can be applied only where there is a complete network.

The modulus appearing in eq (3.9) is the "equilibrium modulus"; the experimental moduli in the work reported in Part I are largely 10-minute moduli. We recognize the distinction, but shall make direct comparisons, since the significant values reported in 
Part I are derived from observations taken under conditions where the creep and set were very small or completely negligible [36, 37]. It has already been pointed out [36] that studies by Thirion and Chasset $[38,39]$ lead to an equation for the equilibrium modulus of natural rubber cross-linked with dicumyl peroxide yielding values differing from the 10 -minute modulus by less than 2 percent when the amount of effective dicumyl peroxide is $1 \mathrm{phr}$ or more.

The insertion of the expression for $\nu_{e}$ from eq (3.8) in eq (3.9) leads to the fundamental equation:

$$
G=\left[2 / 100 M_{d} \bar{v}_{r}\right] R T\left[f p-f w-100 M_{d} / M\right]+G^{*} .
$$

The derivatives are:

$$
\partial G / \partial f \boldsymbol{p}=\left(2 / 100 M_{d} \bar{v}_{r}\right] R T+\partial G^{*} / \partial f p
$$

and

$$
\partial G / \partial T=\left(2 / 100 M_{d} \bar{v}_{r}\right) R\left[f p-f w-100 M_{d} / M\right] .
$$

if one neglects the variation of specific volume $\bar{v}_{r}$ with $f p$ or $T$ respectively.

When $\partial G / \partial T=0$, it can be seen that the cross-linking is such that $(f p-f w)=100 M_{d} / M$ and consequently from eqs (3.10) and (3.7) $G=G^{*}$ and $X / N=1$. Thus all the curves plotted in figure 2 would be expected to pass through a single point independent of the temperature. The coordinates of this point are $f p=f w+100 M_{d} / M$ and $G=G^{*}$. An examination of eq (1.1) and figure 2 shows that the experimental data conform to this surprising prediction, which we have not noted in any previous publications.

At this degree of cross-linking then, each rubber molecule, on the average, is attached to a single added cross-link. This is the "gel point", the degree of cross-linking where the molecules first form a network.

\section{Quantitative Comparison of Predictions and Results}

The equations which have been derived from molecular considerations can now be compared with those found experimentally. In the expression for $G$ given by eq (3.10) the first term has exactly the same form as that given by eq (1.1), while the second term $G^{*}$ corresponds to the sum of $A$ and $H(f p+B)$ in eq (1.1), as demonstrated by eqs (2.1) and (2.3).

The molecular considerations outlined previously, while making allowance for the existence of an energy component, have furnished quantitative results only for the entropy component of the modulus. Consequently they furnish no predictions whatever regarding the value of $G^{*}$. It may be found from the experimental data by inserting the constants found in eq (1.1) into eq (2.3) to obtain

$$
G^{*}=A+H(f p+B)=2.70+0.0684(f p-0.45) .
$$

The experimentally observed term $H(f p+B)$ in eq (1.1) shows that there is a variation of $G^{*}$ with cross-linking and that

$$
\partial G^{*} / \partial f p=H=0.0684 \mathrm{Mdyn}_{\mathrm{cm}}^{-2} \mathrm{phr}^{-1} .
$$

The difference between the solid and the dashed lines in figures 1 and 2, plotting eq (1.2), shows the effect of omitting the term. The value of the term is seen to be quite small under all conditions, and it does not have a very significant effect on the results. The magnitude of the term ranges from -0.031 to $+1.68 \mathrm{Mdyn} \mathrm{cm}^{-2}$ as $f p$ is increased from 0 to $25 \mathrm{phr}$ and of course it is independent of temperature. Its presence does not alter the form of any of the relationships discussed, but causes $G^{*}$, the modulus intercept at $0 \mathrm{~K}$, to increase from 2.67 to $4.38 \mathrm{Mdyn} \mathrm{cm}^{-2}$ as $f p$ is increased rather than remaining constant at $2.70 \mathrm{Mdyn} \mathrm{cm}^{-2}$. The comparison may be readily seen in figure 1 .

It can be seen from eq (3.11) that $\partial G / \partial f p$ should increase linearly with temperature, beginning with a

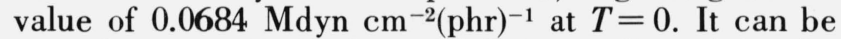
seen from eq (3.12) that $\partial G / \partial T$ is unaffected by the presence of the term containing the constant $H$, as shown by the parallelism of the lines corresponding to eqs (1.1) and (1.2) in figure 1.

The linear relations between $G$ and $T$, and between $G$ and $f p$, as well as the linearity of the derivative curves, are predicted and confirmed by the experimental results.

Probably the most striking prediction of the molecular considerations is that the curves of $G$ against $f p$, for all temperatures, as in figure 2 , should pass through a single point, not located at the origin of coordinates. The theory explains this as the gel point, where the cross-linking is just sufficient to form a network. It is predicted that for this degree of cross-linking $\partial G / \partial T=$ 0 , and at lower degrees of cross-linking $\partial G / \partial T$ should have negative values. These predictions are again confirmed by the experimental values.

A much more severe test of the theory is given of course by a quantitative comparison of the values of the experimental constants $B$ and $S$ in eq (1.1) with the corresponding molecular constants in eq (3.10).

The constant $B$ in eq (1.1) corresponds to the constant quantity $-f w-100 M_{d} / M$ in eq (3.10). Thus

$$
B=-f w-100 M_{d} / M
$$

The first term of this expression, it will be remembered, represents a correction for the peroxide wasted in reactions with impurities in the rubber. The size of this term should depend on the nature and amount of the impurities. Van der Hoff [34] comparing purified and unpurified rubbers found experimentally that $f w$, the amount of this wasted peroxide was $0.31 \mathrm{phr}$ for unpurified pale crepe rubber and that it was independent of the amount of added peroxide up to 4 phr. In the absence of information regarding the 
impurities in our pale crepe rubber we shall assume the same amount as van der Hoff found, namely 0.31 phr.

The comparison of constants now gives

$$
\begin{aligned}
& B=-0.45=-0.31-100 M_{d} / M, \text { and } \\
& \qquad M=27,038 / 0.14=193,000 .
\end{aligned}
$$

This value is a very reasonable one for the effective molecular weight of the rubber before cross-linking. Since the rubber was actually polydisperse, this effective value must represent an average molecular weight, presumably the number-average. No direct measurements of molecular weight were made in the present study.

If the amount of dicumyl peroxide reacting with impurities in the rubber actually has some constant value different from $0.31 \mathrm{phr}$, the calculated value of effective molecular weight will be different from that just given. A value of only 60,100 would have been obtained from the present data if it had been assumed that there were no impurities, so that all the added dicumyl peroxide was available for cross-linking. The calculated molecular weight becomes infinite as the assumed amount of impurity approaches $0.45 \mathrm{phr}$. This possible uncertainty in the molecular weight does not affect any of the other conclusions of the present work.

The second comparison shows that

$$
S=2 R /\left(100 M_{d} \bar{v}_{r}\right)
$$

The values of the gas constant $R\left(=8.3143 \times 10^{7} \mathrm{erg}\right.$ $\left.\mathrm{K}^{-1} \mathrm{~mol}^{-1}\right), M_{d}(=270.38)$, and $\bar{v}_{r}\left(=1.1074 \mathrm{~cm}^{3} \mathrm{~g}^{-1}\right.$, the directly-measured specific volume of the unvulcanized rubber at $25{ }^{\circ} \mathrm{C}$, the midpoint of the temperature range covered) yield a predicted value of $S$ equal to $5.5535 \times 10^{-3} \mathrm{Mdyn} \mathrm{cm}^{-2} \mathrm{phr}^{-1} \mathrm{~K}^{-1}$. The directlyobserved value of $5.925 \times 10^{-3} \mathrm{Mdyn}^{-2}(\mathrm{phr})^{-1} \mathrm{~K}^{-1}$ is about 6.7 percent greater than this.

If this difference is not due to defects in the assumptions, it may arise from the presence of entanglements functioning as pseudo-cross-links and increasing the effective number of sub-chains by about 6.7 percent above that predicted from the actual cross-linking. More precise experiments would be required for a clear-cut decision between these two alternatives. At present, we shall assume that the difference does arise from entanglements and discuss them in a later paper of the present series.

The results of the present investigation yield an experimental value of $\partial G / \partial(f p)$ at $298.15 \mathrm{~K}$ about 10.5 percent greater than that calculated by the simple theory outlined here. About 6.7 percent of this would be due to entanglements and about 3.8 percent to the presence of the term containing the constant $H$ in eq (1.1). Similarly the data of others [40-44] can be put into a form which yields values of $\partial G / \partial f p$ at 298.15 K greater than those calculated here by amounts ranging from about $6-23$ percent.

\section{Conclusions}

The equation

$$
G=5.925 \times 10^{-3}(f p-0.45) T
$$

$$
+0.0684(f p-0.45)+2.70
$$

found in Part I to reproduce the experimental results of time-independent indentation measurements, is represented on a plot of modulus $G$ against crosslinking $f p$ by a family of straight lines intersecting in a single point. The lines have slopes which increase with increasing temperature $T$.

On a modulus-temperature plot, the equation yields a family of straight lines with slopes and intercepts which increase with increasing cross-linking. The intercept obtained by linear extrapolation to $0 \mathrm{~K}$ of experimental data extending from 223 to $373 \mathrm{~K}$ is the component of modulus arising from changes in internal energy on deformation. The intercept represents this component over the range investigated experimentally, regardless of the nature of the actual modulus-temperature relation below $223 \mathrm{~K}$. It exceeds the corresponding component arising from entropy changes at low degrees of cross-linking, specifically when the crosslinking is produced by $2 \mathrm{phr}$ of dicumyl peroxide or less. The energy component predominates over the range where the ultimate elongation and tensile strength have their highest values. With increasing. cross-linking, the ratio of the energy component to the entropy component decreases, since the latter increases linearly while the former remains almost constant.

The entropy component of the modulus as well as its variation with cross-linking and temperature can be predicted by the statistical theory of rubber elasticity, utilizing extremely simplified assumptions. The prediction yields relations of exactly the form found experimentally. Of course, the theory makes no predictions regarding the energy component. As determined experimentally, the energy component is almost constant, increasing only slightly with increasing cross-linking.

The gel point, namely the degree of cross-linking where there is only one effective cross-link per molecule, can be determined experimentally as the degree of cross-linking at which there is no change of modulus with temperature. In our work, this state was reached at $0.45 \mathrm{phr}$ of decomposed dicumyl peroxide, which corresponds to the point of intersection of the lines in the plot of modulus against cross-linking. If the amount of dicumyl peroxide wasted by reaction with impurities in the rubber is estimated, the effective molecular weight of the rubber before cross-linking can be calculated. The modulus at the gel point was found to be $2.70 \mathrm{Mdyn} \mathrm{cm}^{-2}$ at all temperatures in our work. At this degree of cross-linking, just sufficient to form a network, the modulus has no entropy component and consists of the energy component alone.

The experimental value observed for the coefficient of the first term of the general equation for modulus 
as a function of cross-linking and temperature is about 6.7 percent greater than that calculated from the statistical theory, which gives its value in terms of the gas constant $R$, the molecular weight of dicumyl peroxide, and the specific volume of the rubber. This difference can probably be ascribed to the effect of entanglements functioning as pseudo-cross-links.

The third paper of this series will include a discussion of some molecular interpretations, some more sophisticated assumptions, and further conclusions which can be drawn from the present results.

\section{References}

[1] Wood, Lawrence A., Bullman, George W., and Decker, George E., Modulus of natural rubber cross-linked by dicumyl peroxide. I. Experimental observations, J. Res. Nat. Bur. Stand (U.S.), 76A (Phys. and Chem.), No. 1, 51-59 (Jan.Feb. 1972).

[2] Flory, P. J., Principles of Polymer Chemistry, p. 443 (Cornell University Press, Ithaca, N.Y., 1953).

[3] Wood, Lawrence A., The elasticity of rubber, J. Washington Acad. Sci. 47, 281 (1957); Rubber Chem. Technol. 31, 959 (1958).

[4] Cifferi, A., Present status of the rubber elasticity theory, J. Polymer Sci. 54, 149 (1961).

[5] Meares, P., Polymers: Structure and Bulk Properties, p. 173 (D. van Nostrand, London, 1965).

[6] Gee, G., Stern, J., and Treloar, L. R. G., Volume changes in the stretching of vulcanized natural rubber, Trans. Faraday Soc. 46, 1101 (1950); Rubber Chem. Technol. 24, 767 (1951).

[7] Christensen, R. G. and Hoeve, C. A. J., Comparison between theoretical and experimental values of the volume changes accompanying rubber extension, J. Polymer Sci., A-1, 8, 1503 (1970); Rubber Chem. Technol. 43, 1473 (1970).

[8] Penn, Robert W., Volume changes accompanying the extension of rubber, Trans. Soc. Rheology 14:4, 509 (1970).

[9] Allen, G., Kirkham, M. J., Padget, J., and Price, C., Thermodynamics of rubber elasticity at constant volume, Trans. Faraday Soc. 67, 1278 (1971)

[10] Schäfer, E. E., and Rehage, G., On the entropy and energyelasticity of cross-linked high polymers, Makromol. Chem. 123, 91 (1969).

[11] Rehage, G., Schäfer, E. E., and Schwarz, J., Elastic behavior and birefringence in substances with rubber elasticity, Angew. Makromol. Chem. 16/17, 231 (1971).

[12] Paterson, M. S., Effect of pressure on Young's Modulus and the glass transition in rubbers, J. Applied Phys. 35, 176 (1964).

[13] Wood, Lawrence A., and Martin, Gordon M., Compressibility of natural rubber at pressures below $500 \mathrm{~kg} / \mathrm{cm}^{2}$, J. Res. Nat. Bur. Stand. (U.S.), 68A (Phys. and Chem.), No. 3, 259-268 (May-June 1964).

[14] Shen, M., Internal energy contribution to the elasticity of natural rubber, Macromolecules 2, 358 (1969).

[15] Shen, M., Thermoelastic behavior of rubberlike materials at large deformations, J. Applied Phys. 41, 4351 (1970).

[16] Flory, P. J., Cifferi, A., Hoeve, C. A. J., Thermodyıamic analysis of thermoelastic measurements on high elastic materials, J. Polymer Sci. 45, 235 (1960).

[17] Cifferi, A., On the tension-temperature-coefficient of natural rubber, Makromol. Chem. 43, 152 (1961) (English).

[18] Cifferi, A., Hoeve, C. A. J., and Flory, P. J., Stress-temperature coefficients of polymer networks and the conformational energy of polymer chains, J. Am. Chem. Soc. 83, 1015 (1961).

[19] Allen, G., Bianchi, U., Price, C., Thermodynamics of elasticity of natural rubber, Trans. Faraday Soc. 59, 2493 (1963).

[20] Smith, K. J. Jr., Greene, A., and Cifferi, A., Crystallization under stress and non-Gaussian behavior of macromolecular networks, Koll. Zeit. und Zeit. f. Polym. 194, 49 (1964) (English); Rubber Chem. and Technol., 39, 685 (1966)
[21] Krigbaum, W. R., and Roe, R. J., Survey of the theory of rubberlike elasticity, Rubber Chem. Technol. (Rubber Reviews), 38, 1039 (1965).

[22] Barrie, J. A., and Standen, J., Thermoelastic measurements on some elastomers, Polymer 8, (2), 97 (1967).

[23] van der Hoff, B. M. E., and Buckler, E. J., Transient changes in topology and energy on extension of polybutadiene networks, J. Macromol. Sci.-Chem. Al(4), 747 (1967).

[24] Shen, Mitchell C., McQuarrie, Donald A., and Jackson, Julius L., Thermoelastic behavior of natural rubber, J. Applied Phys. 38, 791 (1967).

[25] Boyce, P. H., and Treloar, L. R. G., The Thermoelasticity of natural rubber in torsion, Polymer 11, (1), 21 (1970).

[26] Tanaka, T., Yokoyama, T., and Yamaguchi, Y., Methods for the determination of internal energy contribution to rubber elasticity, Memoirs, Faculty of Engineering, Kyushu University 29, 89 (1970); Rubber Chem. Technol. 44, 127 (1971).

[27] Shen, M., and Blatz, P. J., Energy contribution to rubber elasticity, J. Applied Phys. 39, 4937 (1968).

[28] Guth, E., Recent developments in the statistical mechanics of polymers, J. Polymer Sci. C3 1, 267 (1970).

[29] van der Hoff, B. M. E., Relation between energy contribution to elastic force and strain dependence of modulus for polybutadiene vulcanizates, J. Macromol. Sci.-Chem. A5, (4) 661 (197i).

[30] Meares, P., Polymers: Structure and Bulk Properties, Chapters 6, 7, and 8, pp. 160-236 (D. van Nostrand, London, 1965).

[31] Flory, P. P., Principles of Polymer Chemistry, Chapter XI, pp. 432-494 (Cornell University Press, Ithaca, N. Y., 1953).

[32] Treloar, L. R. G., The Physics of Rubber Elasticity, 2d ed. (Oxford University Press, London, 1958).

[33] Meares, P., Polymers: Structure and Bulk Properties, p. 203 (D. van Nostrand, London, 1965).

[34] van der Hoff, B. N. E., Reactions between peroxide and diolefins, I. \& E. C. Product Research \& Development 2, 273 (1963); Rubber Chem. \& Technol. 38,560(1965).

[35] Bristow, G. M., Moore, C. G., and Russell, R. M., Determination of degree of cross-linking in natural rubber vulcanizates. VII. Cross-linking efficiencies of di-tert. butyl and dicumyl peroxides in the vulcanization of natural rubber and their dependence on the type of rubber, J. Polymer Sci. A3, 3893 (1965).

[36] Wood, L. A., Creep of "pure-gum" vulcanizates of natural rubber, J. Rubber Res. Inst. Malaya 22 (3), 309 (1969); Rubber Chem. Technol. 43, 1482 (1970).

[37] Wood, L. A., and Bullman, G. W., Creep and other tensile properties of rubber cross-linked by dicumyl peroxide, J. Polymer Sci. A-2, 10,43 (1972).

[38] Thirion, P., and Chasset, R., Relative contributions of viscoelasticity and aging to the relaxation of rubber vulcanizates, Proc. 4th Rubber Technology Conference, London, May 1962, p. 338 (Inst. of Rubber Ind., London, 1963); Rev. Gen. Caoutchouc 41, 271 (1964); Rubber Chem. \& Technol. 36, 50 (1963).

[39] Chasset, R., and Thirion, P., Viscoelastic relaxation of rubber vulcanizates between the glass transition and equilibrium, Proc. Int. Conf. Non-crystalline Solids, Delft, 1964, J. A. Prins, Ed., p. 345 (North-Holland Publishing Co., Amsterdam, Interscience, New York, 1965); Rubber Chem. Technol. 39, 870 (1966); Rev. Gen. Caoutchouc 44, 1041 (1967).

[40] Moore, C. G., and Watson, W. F., Determination of degree of cross-linking in natural rubber vulcanizates. II. J. Polymer Sci. 19, 237 (1956)

[41] Mullins, L., Determination of degree of cross-linking in natural rubber vulcanizates. III. J. Applied Polymer Sci. 2, 1 (1959); Rubber Chem. Technol. 34, 279 (1961).

[42] Mullins, L., and Thomas, A. G., Theory of rubber-like elasticity, Chapter 7 in book, The Chemistry and Physics of RubberLike Substances, L. Bateman, Ed. (Maclaren, London, 1963).

[43] Redding, R. B., and Smith, D. A., Rapid measurement of the chemical contribution to network chain density for natural rubber, J. Polymer Sci. C30, 491 (1970).

[44] Treloar, L. R. G., Introduction to Polymer Science (Wykeham Publications Ltd., London, Springer-Verlag, New York, 1970).

(Paper 77A1-761) 


\section{UNITED STATES}

GOVERNMENT PRINTING OFFICE

PUBLIC DOCUMENTS DEPARTMENT

WASHINGTON, D.C. 20402

OFFICIAL BUSINESS

U.S. GOVERNMENT PRINTING OFFICE POSTAGE AND FEES PAID 375

PENALTY FOR PRIVATE USE, $\$ 300$

JOURNAL OF RESEARCH of the National Bureau of Standards

Vol. 77 A, No. 1

Jan.-Feb. 1973

A. Physics and Chemistry

\section{Contents}

Page

Spectrum and energy levels of neutral thulium. J. Sugar, W. F. Meggers, and P. Camus.

Simultaneous measurement of specific heat, electrical resistivity, and hemispherical total emittance of niobium-1 (wt. \%) zirconium alloy in the range 1500 to $2700 \mathrm{~K}$ by a transient (subsecond) technique. Ared Cezairliyan.

Theory of disclinations: II. Continuous and discrete disclinations in anisotropic elasticity. R. deWit.

Photochemistry of methane in the photoionization region. R. E. Rebbert and P. Ausloos.

Radiolysis of methane. Formation of acetylene. R. E. Rebbert and P. Ausloos.

The enhancement of water vapor in carbon dioxide-free air at 30,40 , and $50{ }^{\circ} \mathrm{C}$. Richard W. Hyland and Arnold Wexler.

The second interaction (cross) virial coefficient for moist air. Richard W. Hyland and Arnold Wexler.

Solubility and surface properties of finely divided hydroxyapatite. Y. Avnimelech, E. C. Moreno, and W. E. Brown.

Pyrolysis of anionic and thermally prepared polystyrenes. Leo A. Wall, Sidney Straus, Roland E. Florin, and Lewis J. Fetters.

Modulus of natural rubber cross-linked by dicumyl peroxide. II. Comparison with theory. Lawrence A. Wood. 